\title{
Interview
}

\section{Reviewing the innovations of Cumulus 8 - An interview with Uli Knocke of Canto, Incorporated}

\section{Ulrich Knocke}

joined Canto as Product Manager in January 2001, later assuming the position of VP of Product Development. In 2006, Knocke took the reigns of Canto as CEO, Incorporated. Ulrich Knocke graduated with a degree in Computer Science from the Technical University of Karlsruhe, Germany, while working for Apple Computer. Before joining Canto, Knocke served as Product Manager for Hermstedt AG.

\section{ABSTRACT Michael Moon interviews Uli Knocke of Canto, on the recent major upgrade of their very popular Cumulus DAM system.}

Journal of Digital Asset Management (2009) 5, 159-176. doi:10.1057/dam.2009.5

Keywords: Cumulus; tagging; dam; metadata; archive; server

MM: We're here with Uli Knocke of Canto discussing the major upgrade of their very popular Cumulus DAM system.

UK: First, let me thank you for the opportunity to bring your readers up to speed on Cumulus. We're pretty excited about it.

MM: However before we start, Uli would you give us a bit of your background, starting with your current position?

UK: I've been Canto's CEO since August of 2006. Before that, I was Vice President of Product Development. I started with Canto back in January of 2001. I hold a degree in computer science from Technical University of Karlsruhe. During my university time, I worked for Apple Germany. Prior to joining Canto, I worked for an Apple reseller, and was product manager at Hermstedt. I've always worked for internationally active companies, and I've seen business from the user, reseller, partner and vendor perspectives. I'm in my final steps now of finishing my MBA Entrepreneurship degree to complement my technical skills.

MM: What's the corporate history of Canto?

Correspondence: Ulrich Knocke, CEO 221 Main Street,

Suite 460, San Francisco, CA 94105, USA

E-mail: Info@canto.com
UK: Canto was founded in 1990. The first product was scanning software called Cirrus, which explains the lineage of the Cumulus brand name. Cirrus was popular, but it was totally overshadowed by Cumulus, when the original version shipped a few years later. It was clear to Canto that Cumulus was its future. The company has always been privately held, which has enabled us to follow our own lead with regard to our evolution. I think that's also one of the main reasons we've been able to last this long, and stay on top of the pack the entire time, with regard to sales.

MM: Okay, so how many Cumulus systems has Canto sold?

UK: According to our records, we've sold over 13500 Cumulus Servers and shipped more than 1150000 user licenses.

And I think those sales are attributable to a few things. First and most obvious, we've sold DAM systems for much longer than most other companies; we had quite a head start. More than once I've heard people refer to Canto as the inventors of the DAM market! But I think product has a lot to do with it too. Cumulus has always been priced far more affordably than other DAM systems, especially when you consider all it can do. Plus, Cumulus is both a product and a platform. It integrates very easily into other infrastructures, so it's attractive to users, integrators and development partners. MM: With numbers such as those, Canto clearly maintains a dominant market share. Do you intend to become the Microsoft of DAM? 
UK: [Laughs] Are you trying to get me into trouble with our Mac user base? Sure, you could compare Canto with Microsoft when you think of dominant market share; however I think there's one very important difference: one of Canto's primary goals in all our development is to break down the barriers between computing environments. You know, offer users a common experience no matter which platform they prefer - Windows, Mac, Linux, Solaris. Understandably, that's not a goal for Microsoft.

Microsoft is the default choice for most computer users, as worldwide market share shows, but it's not the best choice for everyone or for every purpose. And, while Cumulus is the default $D A M$ choice for most organizations for what I think is good reason - our situation differs from that of Microsoft. We're smaller, more agile and dedicated to doing one thing brilliantly and not many things just okay.

MM: So, let's talk about your customers? Who are they?

UK: Are we looking for industries? Sure, we have a huge customer group in print/publishing houses and ad agencies. But, even though our roots have grown from those industries, we are most successful today in manufacturing, government and education. We have good success stories in the medical sector as well. And you can see many common use cases across these industries, from archiving, to corporate communication and marketing usage, to distribution of files to all kinds, to workflow assistance, such as approvals.

MM: Manufacturing, government and education describe segments that are quite diverse. What are the common needs among those groups?

UK: I can identify what I think are the key aspects of Cumulus that attract those groups, but I don't believe it's always the same thing. On the manufacturing side, customers are looking for ease of use, and ease of integration with other systems, such as inventory and accounting. On the education side, cost is a huge consideration, but they also tend to want as much flexibility band for their buck as they can get - their budgets are limited, but their knowhow and needs are anything but! Government is also budget conscious, particularly in these times, but security is extremely important to them, something that has always been strong in Cumulus.

MM: Okay, what we're here to talk about today is Cumulus 8, Canto's latest release. Before we get into details, I'd like you to give me a high-level summary of what's new in Cumulus.

UK: On the user side of things, we've done a lot of important fine-tuning, from the user interface (UI), to the help system, and much more. Search options have been improved to help users find what they need faster, and we've added some great new cataloging technologies. We also added a built-in image editor that lets users crop, blur, format-convert and many other things - even from within the Web Client.

Less visible to users - but something they'll definitely recognize - the communication between the Cumulus Client and Server is much more efficient now, so they'll see searches and other things they use all the time start taking far less time. Plus, the Native Client is fully multi-threaded now, so users with multicore machines are going to think they've died and gone to Cumulus performance heaven! And, in addition, we're now offering asset tracking and statistics, which you can think of as something like Google Analytics for DAM. DAM is not just about finding things anymore; it is also about providing tools to our customers that help them optimize their tagging, etc. Now they have a full toolbox to see what their customers are searching for, what assets are downloaded the most, which queries did not result in results. All that can be invoked instantly, but also automated - every week the marketing director can get the latest statistics and reports. And with annotations, we support collaboration between users and support their workflow even more.

Overall, Canto spent a long time listening to users and partners, and we spent a long time testing everything that's new. In a way, you can't look at Cumulus 8 as being an upgrade from Cumulus 7 - it's more like a precursor of things to come in future versions. Sure, users will see a better looking UI today, and things will work better and faster - and even easier but what they don't know yet, is that when they upgraded to Cumulus 8 , they upgraded to an entirely new generation of Cumulus.

MM: What about on the back end? 
UK: On the lowest level - the application programming interface (API) - we've essentially started from scratch, while retaining absolute backward compatibility. You might say that our transition from script-based technologies is complete, and we now offer a completely modern, programming interface that Java and $\mathrm{C}++$ developers will absolutely love.

MM: What is it they will love, and why didn't you do whatever you've done now, before?

UK: First off, everyone gets nervous when you talk about an API change. No company likes to do them, because they can be expensive in terms of getting developer support, and retraining. But we knew it was time, and we did this carefully, so that usually nothing more than minor tweaking would be required to start taking advantage of the newer code and methods. Our developers are already very happy about that!

Plus, they love it because we're offering pure development environments that are not based on older technologies, like AppleScript or OLE. We can, of course, still interface with those technologies, but everything is more modern now. Before, we let partners develop in, say, Java, but we sometimes made them 'think' in terms of the way things would have been done in those old days or using those old technologies. So, in letting developers work now with Cumulus the way they have been asking, they get to take advantage of their core development knowhow, instead of worrying about the way Cumulus developers did things 10 years ago.

And, because we've cleaned it up so much, the performance improvements are huge when developers take advantage of the new options.

MM: What's the learning curve like for existing developers?

UK: I can't imagine any of our developers having any trouble getting up to speed fast. What we've done is that we've gotten out of their way - given them an environment more like they're used to as developers. Instead of saying, 'Hey, we're Canto and this is how we see Java,' we're giving them a pure Java environment from which they can think like Java developers. In February, we held a 'Coding Kitchen' in Berlin. We had Cumulus partner developers from all over the world attend.
I would say the 'excitement curve' completely overshadowed any learning curve! Plus, they were so thankful that we did in plenty of time for them to update their applications to take advantage of the new possibilities Cumulus 8 offers them.

MM: Cumulus 8 sounds almost like a complete overhaul of the product. Is that fair to say?

UK: Well, it is still Cumulus, so users and administrators who use Cumulus now will not be lost. But, what's going on under the hood is very different.

MM: Why now? Why did you decide that Cumulus 8 needed all this work, as opposed to, say, Cumulus 6 or Cumulus 7?

UK: In many respects, what users saw in Cumulus 7 was an incremental update to Cumulus 8. We've known this technology overhaul was coming for many years, and we knew it would take time to perfect; but we also knew that we couldn't stop shipping product in the meantime. So, as new technologies were built, we seeded them in Cumulus 7 updates. Now, with Cumulus 8, we're activating all those technologies to really start delivering value to a wide range of users.

MM: Uli, I propose that we walk through the basic structure of the Cumulus DAM software and related capabilities, starting with the UI. What kind of changes have you made to UI in Cumulus 8?

UK: We gave it a newer, more contemporary feel - new icons, a more modern, streamlined appearance. Now, it's not an entirely new interface, though - you would still recognize it as the Cumulus Client. We added the ability for users to interactively resize thumbnails using a slide bar. This is really handy when you want to precisely fit a collection of thumbnails on your screen. And it's fantastic when you've cataloged large thumbnails, because you've got what amounts to an interactively resized preview. There are also a slew of new context-sensitive help options that will help users sort out things like why a query returned no results.

MM: How might these changes require additional user training?

UK: We believe that all of our current users will continue to work as they do now. Some of 
the newer features won't even be obvious to them. Things like the thumbnail scaling will be obvious and easy to understand though. Most of the improvements, though, will become apparent only after working with Cumulus 8 for a while; once you really get into it. Every now and then, users will notice some small tweak that makes the UI more usable or attractive. MM: In the past, users have criticized Canto for not having a friendly UI in Cumulus. How have you made Cumulus 8 more in step with other applications and ease of use?

UK: Let's start with a basic observation: many 'very friendly' UIs mask the fact that the application has a lot less functionality. Once you get into a certain class of application, you have to expect additional complexity. Someone can also develop a DAM system that can do less of what Cumulus can do; there you can get away with less gadgets and options in the UI. We have a simple idea behind the UI design in Cumulus: offer easy access to the features that users need most of the time, without hindering access to the rest.

MM: Photoshop comes to mind as an example of a complex application that fits that paradigm you describe.

UK: Photoshop is a great example. That UI offers far more complexity than the Cumulus UI, but users have grown to know and respect the program. Cumulus is the same. Ask someone who uses Cumulus every day, and she'll find it no more complicated than someone who uses Photoshop finds that application.

MM: Yes, and there's Photoshop Elements for those who can't come to terms with Photoshop. Perhaps Canto should offer a 'Cumulus Elements'?

UK: Photoshop Elements might be easier to understand at first, but it's definitely not Photoshop. And, actually, there is sort of a 'Cumulus Elements,' but its made by one of our competitors, if you know who I mean. And it's even more expensive than Cumulus!

MM: Let's talk about some of the administration and maintenance changes for Cumulus 8. Will Cumulus administrators welcome the version 8 and why?

UK: Without a doubt! We added or enhanced many things in Cumulus 8 that with easier administration in mind. Let's start with performance.
UK: The performance of Cumulus 8 across the board will become favorite enhancement.

Administrators will particularly like that we now take better advantage of current advancements in hardware. We've adapted Cumulus for multicore processors; our thread-handling architecture sucks up as much of the added performance offered by these multi-core processors.

Cataloging assets and updating data goes much faster (cataloging is Cantos term for importing new files into the database/catalog). It's almost unfair to even compare Cumulus 8 to earlier versions!

MM: So, no spinning wheels or hourglasses?

UK: Well, fewer spinning wheels - we can't get away from spinning wheels or hourglasses entirely. But when the hardware's in place to better handle the heavy lifting, we're now taking better advantage of it.

MM: But Cumulus, to my knowledge, has been multi-threaded for a while. What's different now?

UK: Previous versions of the Cumulus Server were multi-thread aware, because multi-core processors were more common on servers, so we took advantage of it. But the Cumulus Client software was not taking advantage of multi-threading until now. Starting with Cumulus 8, pretty much everything is multithreaded - cataloging new assets, updating asset records, assigning metadata - you name it. MM: So now, as dual- and quad-core microprocessors become more popular, the Cumulus 8 Client is taking advantage of some of their built in simultaneous processing capabilities?

UK: Yes, and this is great news for users, because much of the workload of cataloging and processing assets is handled by the Cumulus Client software (Figure 1). That's all much, much faster now, thanks to our friends at Intel and AMD - and, of course, some folks at Canto too!

MM: I assume the performance enhancements are cross platform?

UK: Absolutely. There once was a time when our Windows Client had a solid performance edge over the Mac, because of the difference in processors. But Cumulus running on an Intel Mac is like an entirely different universe from running it on a G5, or anything older. 

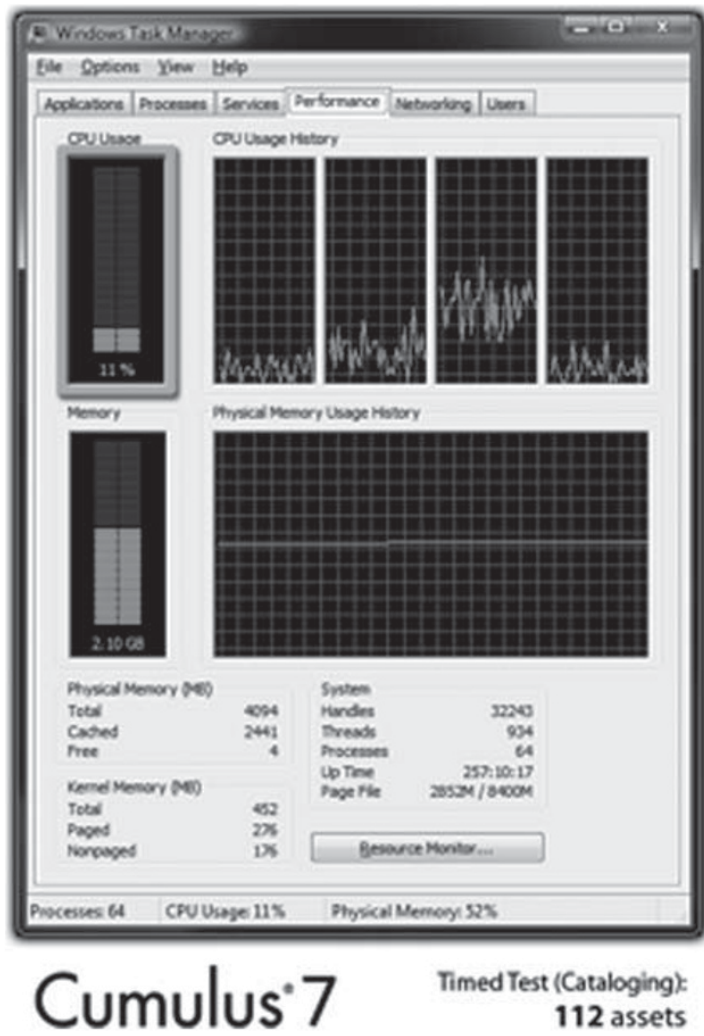

Timed Test (Cataloging): 112 assets

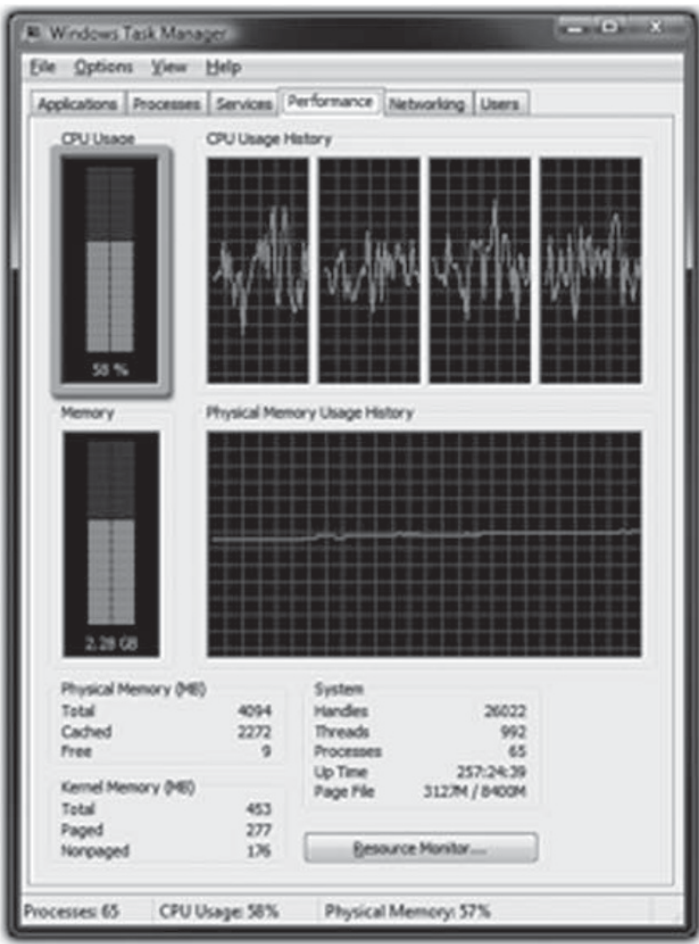

Timed Test (Cataloging): 567 assets

Figure 1: Cumulus 8 offers multi-threaded performance that takes advantage of multi-core processors and multi-processor machines for significant performance boosts for both the Cumulus Client and Cumulus Server. This test pitting the Cumulus 7.6.1 Client against an alpha test version of the Cumulus 8 Client. Highresolution thumbnails were created for each asset (1024 pixels), and the test was allowed to run for a minute. A Cumulus 8 Server was used, giving both Clients an increased performance edge over a Cumulus 7 Server.

MM: The Cumulus Server is available on several platforms. Is any server platform a clear winner for you performance wise?

UK: No, and I'm not just saying that to be nice! The core code Cumulus is built on is used across all our Servers running on Windows, Mac OS, Linux and Solaris. And since all those operating systems are basically running on the same hardware these days, we're seeing comparable results across the board.

MM: Anything else new in the performance department before we move on?

UK: We've changed the API, which results in many things working faster, such as assigning categories. You wouldn't notice a speed bump here if you assigned one asset to one category. But if you want to assign 500 assets to a handful of categories, making all those connections at once, you'll find Cumulus 8 magnitudes faster than previous versions.
Also, the Cumulus Client is 'talking' to the Cumulus Server more efficiently now, so things like asset record scrolling are much faster. That's because information not needed by the client isn't transferred.

MM: What about the Cumulus administrator's workflow? Any improvements there?

UK: One new thing that we've got is catalog templates. You've decided what works for your catalog - you have fields; you've set up properties for the fields; you've done all these things. Now, in Cumulus 8 , you can save that entire configuration as a template.

So you, or another division of your company, or whomever, can actually use a clone of your catalog, based on that template.

MM: So this allows me to create a bestpractice template, of sorts, that I can propagate through my work groups and teams. Shall I 
assume I can also then apply certain permissions and policies to who gets to use the templates?

UK: That's exactly right. Any permissions that are specific to the catalog fields are copied along with it. But asset permissions, obviously are not, because the catalog templates include no assets. It's just a nice little thing that enables customers to actually rubber-stamp their entire process.

MM: Access to assets the catalog will contain is not saved as part of the template?

UK: No, because asset access controls in Cumulus are based either on user accounts or the assets themselves. Catalog templates contain access restrictions for things that are inside the catalog structure itself, such as who can edit a given metadata field.

MM: So a catalog template will not let me determine who can access the catalog or what they can do with the assets cataloged into that catalog?

UK: No, but we do have some user management improvements in Cumulus 8 that make setting and understanding user permissions easier.

MM: Would you explain?

UK: Sure, there are definitely areas that have been improved here, including a new simplified user management mode, which makes it easy for admins to determine permissions for the most common things, like read/write access, which catalogs, etc.

Then, from within the Cumulus Preferences window, you can see a summary of all the permissions available for an open catalog, and for shared Cumulus Server resources, such as views, asset handling configurations and asset processing actions. There's never been a really fast way like this to see a globally summary of who can do what, so administrators are going to love this.

MM: How does the simplified user management work if the system is already configured? Is it compatible with the previous permissions model?

UK: It actually is the previous permissions model; we're just offering a new interface to it. Experienced Cumulus admins can use the previous interface if they like, or they can also use this one - it's not an either/or thing, both work all the time.
MM: Is access to the permissions summary that's inside the Client available only to administrators? Or can users see their own permissions?

UK: Access to this screen is actually part of the Catalog Setting permission, which is a standard Cumulus permission that can be granted to other users besides the administrator. By default, only the Cumulus administrator will see it, though.

MM: Let's examine Cumulus as an innovation platform. How does this platform speed innovation to internal customers or to a market, highlighting the idea of incremental or progressive upgrades to individual users, workgroups or permissions? In your answer, I'd like you to address the challenge of traditional enterprise applications: if you do an upgrade, everyone has to get upgraded whether they need or want to upgrade. Or it means that you have to install a bunch of new clients, such as Microsoft Office. If you've got 50-100 or 200 or 1000 , that could be quite a task. Either way, you've got a whole lot of things in motion that could result in problems. Right?

UK: Cumulus has some core strengths in all those areas. The first relates to the development and upgrade of the customer's catalogs. With Cumulus, you never have to take the system offline in order to make changes to a catalog, even substantial changes like add and deleting metadata fields or changing permissions. You never have to disrupt or disconnect clients when you're making these sorts of changes, which is fantastic for production environments.

From the customer's perspective, this means they don't need to spend months planning their initial deployment, holding up their ROI all the while. They can install the 'shrink-wrapped' product, get it running in a few minutes and start grabbing metadata. There's no reason not to!

As an example, even if a catalog they launch with lacks some metadata fields they'll need to capture everything they want from their assets, and they realize later, 'Oh, no! We haven't been grabbing all the EXIF metadata!' there's no problem. They just add the fields they need, and update the records already in the catalog - a single step process. Cumulus will go back through the original assets and grab the missing data. 
MM: And this can happen without taking the system down?

UK: Absolutely. Connected users wouldn't even know it's happening. They'd just all of a sudden start seeing additional metadata. It's great because you never need to worry that you'll lose data if you don't plan everything up front. Cumulus is flexible enough to always let you rethink the way you do anything. That's a great advantage.

Plus, it enables customers - administrators and users alike - to start out easy. And, as they gain more familiarity with the system, they can start to take advantage of its more sophisticated aspects.

MM: So what about Cumulus software updates themselves? Surely you must take the system down for those. Right?

UK: Yes, but even that can be done with minimal disruption, because we don't require simultaneous server/client updates.

Administrators can take the Server down, update it - which like the initial installation is a wizard-based, double-click process that takes only a few minutes - and then update the Client software when it's convenient. You could do the entire thing over a weekend, or you could even update the Server over lunch it's really an easy process.

For some upgrades, an update of catalog files might be required, because of architecture updates. In the vast majority of updates, it's not necessary, but even when it is, we provide a menu option to make it easy.

MM: Will Cumulus 8 require catalog updates?

UK: Yes, because we've got some major architecture advances in there. But, as an example, the move from Cumulus 6.6 to Cumulus 7.0 was the last time we required catalog updates, and that was several years ago. But the migration work is handled by Cumulus, so there's nothing a catalog admin needs to know other than a conversion will occur as soon as they open the catalog in Cumulus 8 . MM: Getting back to the software updates, what happens to my Cumulus 7.6 Clients if I update the Cumulus Server to version 8? UK: Say you performed the Server upgrade over the weekend. Come Monday morning, your users fire up their Cumulus 7.6 Clients, and they get to work, just like they ordinarily would. They would have no idea that the back end has completely changed.

MM: Would this mean that if I had Cumulus 7 and/or maybe 6 or 7.6 Clients out there (because they're at remote offices or traveling), I could upgrade my Cumulus 8 Server and have older versions of end-users clients still access the upgraded server? And for all intents and purposes, would that mean getting the same services they got last week?

UK: Exactly. The only thing they might do is call you and say, 'Michael, did you just buy new hardware? This is a lot faster than it was last week!' They might notice that!

MM: How many versions of the client do you go back and support on the 8 Server?

UK: We'll support clients as far back as Cumulus 7.1.1. Cumulus also includes a setting that enables an administrator to choose which Client versions users can actually connect with. So, if you're trying to filter out, say, your 7.0 and 7.1 Clients, you can actually go in and tell Cumulus, 'Stop letting 7.0 Clients connect.' When users connect with those old Clients, they get a message to contact the administrator.

This is particularly great at bigger installations, where it's tough to know who's got 7.0, 7.1, 7.5, 7.6, 7.6.1. So, Cumulus sort of helps you pull up the rear by incrementally discontinuing your own support.

MM: I wouldn't have to do that across all my 7.0 s? I could do the $7.0 \mathrm{~s}$ in this particular department or class of user?

UK: Yes, because the setting is an aspect of user permissions.

MM: That means I could turn one guy off.

UK: Yes. Or, if you were using groups-based permissions, you could disable an entire group. And there are important advantages to being able to control how or when users connect. For example, you might want to prevent a group of users from using the new Cumulus 8 Client until you've had a chance to go over it with them. So, even if they connect from someone's machine who has the Cumulus 8 Client installed, it won't work on their account until you make it work. $\mathbf{M M}$ : Any updates to the ways in which users can catalog their assets?

UK: Yes, Cumulus 8 includes some very strong new cataloging options. First off, we've introduced a brand new metadata pre-filler that works just like users always wanted. 
MM: Cumulus already included a pre-filler, right? So what's new with this one?

UK: Before, we let users enter a few notes and make category assignments. But they always asked for more options. So, with Cumulus 8, the pre-filler works just the way users always wanted it to work. When it appears, what you see is a view of metadata fields that you define specifically for this purpose. So, you can choose which fields should be included.

MM: So I can choose which fields appear from any of the catalog fields available?

UK: Yes, and you can choose any or all fields. So, for example, say your catalog includes 20 metadata fields, but you need to have five of them filled in when the assets are cataloged. Now you can choose those five fields to appear in the pre-filler.

Even better, because access is determined by permissions, you can present a pre-filler form that makes sense for the connected user. Say, for example, you want your customers to be able to upload assets without forcing them to enter lots of metadata, but you require your employees to not be so lazy!

MM: Can I force the entry of metadata into certain fields?

UK: Yes, that feature has been in Cumulus for a while, but it becomes even more useful now, because you can make sure your mandatory fields appear in the pre-filler.

MM: What else is new?

UK: In a way, we've opened up the conventional definition of cataloging in that you no longer need to have access to a Cumulus Client in order to get your assets into Cumulus. Cumulus 8 makes it possible to catalog via email or from 'drop folders' on file servers.

This is not only a convenience for remote users, it's a benefit for catalog administrators, because you don't always want to give client access to everyone who needs to catalog new assets. It's much easier to just give them an email address and tell them to attach the files. Everyone knows how to do that.

MM: How exactly does this work? I just attach files send them off?

UK: That's about it! The POP3 email account you use acts as a staging area for assets to be cataloged. Cumulus pings the account at certain intervals and retrieves everything it finds. It catalogs each email message, including the attachments, which it links to the messages themselves. So, if you want remote employees or customers to be able to catalog, you just tell them to email the files to something like CatalogMe@MyCompany.com.

This is a great new feature for everyone, but imagine how this opens up Cumulus to users with camera phones. Real estate agents taking photos of houses, or insurance adjusters getting claim shots can just email their photos to Cumulus (Figure 2). Think about it: take a picture with your smart phone camera, send it via your mobile network, and Cumulus catalogs it.

But this also opens Cumulus up to nonhuman clients, such as security cameras that take snapshots every minute or so. They can be configured to email those shots to Cumulus, where they are cataloged, time stamped and immediately available to others, no matter where the camera itself is located.

MM: That would also probably apply to some of these WiFi-enabled cameras. Find an available WiFi link and send it off someplace. Right?

UK: That's a great example too. Another example is compliance. For example, a new German law requires companies to archive all their email. Now, companies can use Cumulus for that purpose, in addition to all their other DAM needs. You just configure your corporate server so that any e-mail sent via your network is automatically cc'd on the back end to your Cumulus email address. A company's entire e-mail stream - incoming and outgoing - now can be cataloged into a Cumulus database.

MM: So, as e-mail gets checked in to a Cumulus catalog, I'm just going to find a little 'e' for the thumbnail, indicating it was an e-mail? Or am I actually going to see the text of the e-mail in the thumbnail?

UK: You can use whichever image you'd like to act as the thumbnails for catalog email. But, no, you won't see the email contents in the thumbnail. Inside the information view, you'll see the actual text of the e-mail and you'll see the attachments. This is great because the sender can provide details that can be copied into the appropriate metadata field.

MM: So, then, you could probably copy/past text from the e-mail?

UK: Sure. Yes. That's all accessible. 


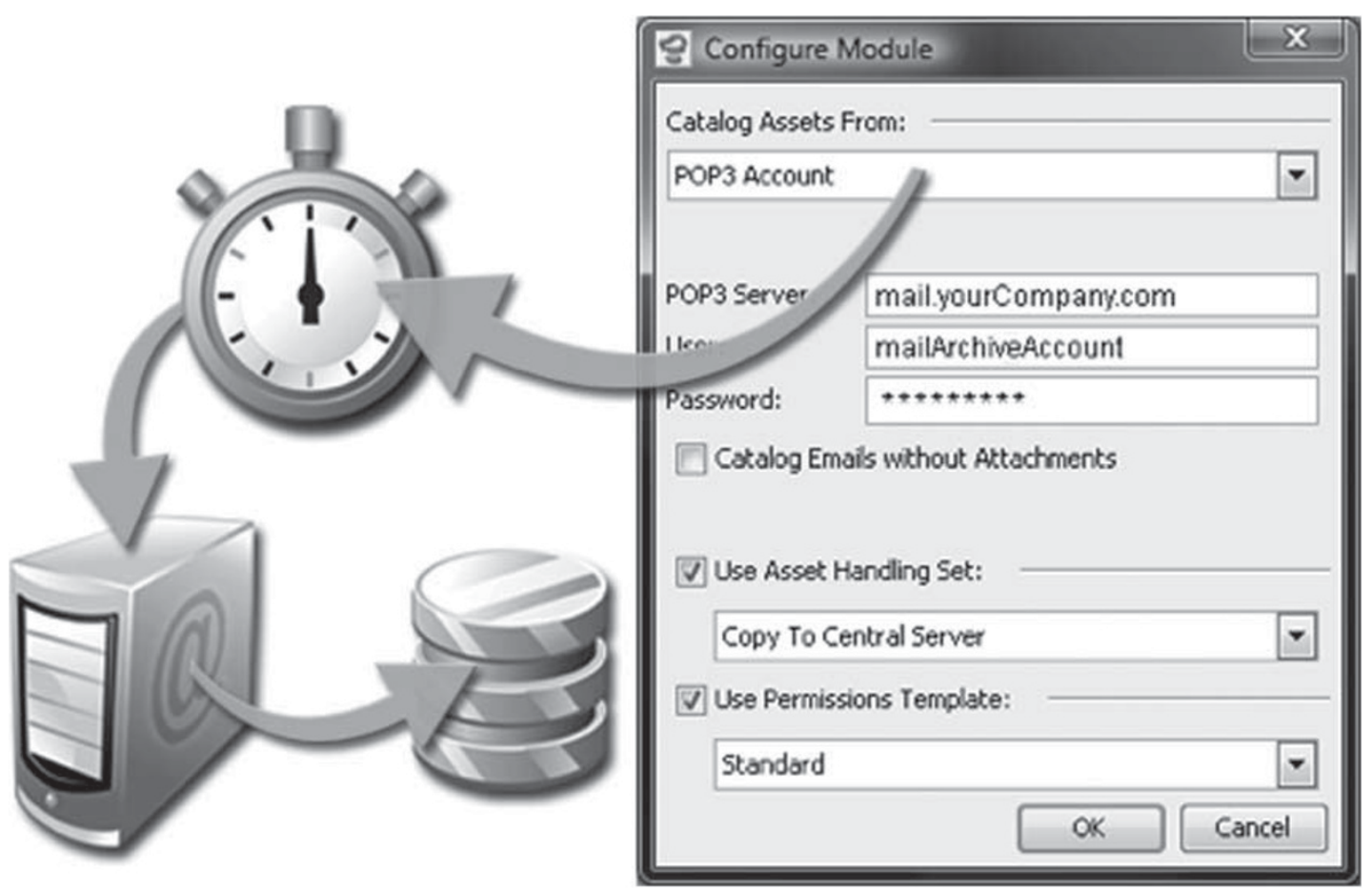

Figure 2: Cumulus 8 makes it easy to catalog and archive email, including any attachments, at schedules users define. Autocataloging from a file system location can be configured the same way.

MM: Any other cataloging options we should know about?

UK: Yes, Cumulus 8 also makes it possible to define a local file location to be used for cataloging. What's new here is that we're doing it on the server side, so it doesn't require the Cumulus Client software.

MM: Like a watch file or hot folder? So if anybody puts a file or project into the drop folder, Cumulus 8 then takes that as a submission and puts it into the queue for the asset specialist to formally catalog it, applying all the right metadata and policies?

UK: Yes. The modular approach of Cumulus enables to grab files from a Mac, PC, Unix workstation, or even from an FTP location. The Cumulus Scheduler is used to determine the intervals at which cataloging occurs.

MM: So, if a customer wants to accept usergenerated submissions, I could support any number of my users to a photo, PDF using a standard browser to some FTP submissions queue wherever ... out there in the DMZ or on the public Web?

UK: Yes, and I can give you a real-world example of a customer doing this. The
Hamilton Public Library in Canada not only archives historical photos from their region, they now encourage the public submission of photos. Traditionally, the submission process represents a big stumbling block for libraries. What's the best way to get assets and enable submitters to provide information about them? With Cumulus 8, Hamilton can publish a submissions e-mail address and say, 'Send us your historical photos! In the e-mail, explain what it is, what you did, who you are and all that stuff.' Cumulus 8 ingests the photos, grabs the email text and treats it as metadata - albeit unstructured text with potentially lots of misspellings.

Nonetheless, this speeds the collections process by orders of magnitude, adds new value to their collection and increases levels of engagement within their community. Plus, along with the assets, they get an email address of the submitter, which enables them to follow up if they need more info, or a release.

MM: Anything else on cataloging before we move on?

UK: Well, sort of. One long requested feature has been the ability to create an asset record before the asset is cataloged. So, for example, 
you can create asset records for the assets you expect from a photo shoot or other project, long before the assets themselves even exist.

MM: So they act as placeholders?

UK: Yes, but even better, Cumulus 8 makes it much easier to really maintain control over project management, because you can now track things that haven't yet been cataloged. So, if an asset was due on, say the 15th, and it's now the 20th, you'll know it's missing. In fact, the Cumulus Scheduler could even be used to send out reminders. Before Cumulus 8, there was no easy way to keep tabs on expected assets.

MM: Have any search technologies been added or improved with Cumulus 8?

UK: The Cumulus metadata engine has always been optimized for metadata management, so we often outshine conventional database systems in terms of sheer speed. Now, with our Clientside multi-threaded performance boost, we can take some of that search power from the Cumulus Server and move it onto the desktop. For example, in previous versions of Cumulus we introduced the concept of dynamic, rulesbased permissions in a feature we call Live Filtering. Now, we've taken that same real-time search technology to the desktop as 'User Live Filtering.'

MM: So, what does User Live Filtering actually mean for users?

UK: User Live Filtering is like adding a layer of search criteria on top of whatever other search criteria you use. So, for example, say you've got 100000 records in your catalog. You're trying to find a selection of screenshots to e-mail to a press outlet. There are certain search criteria that would be considered constant for that purpose, such as file format - you're not looking for PowerPoint or InDesign files you're looking for JPGs or PNGs. User Live Filtering enables you to define those constants so you can better focus on the details of what you need.

MM: So I could define my preferred file formats in the filter and Cumulus would show me only files of those types?

UK: That's exactly correct. But your filter can contain any metadata. For example, you might want to only see stuff rated by four or five stars. Or, maybe just approved assets. Another example would be limited the asset records you see to only those associated with a certain project or client you're working on. You just activate a user Live Filter and continue using the catalog as you normally would, knowing everything you see is within the limits of the filter you've defined.

MM: Could I force a user live filter on a certain user or group, say, to make sure that only approved assets are available to them?

UK: Well - yes and no. 'No,' because User Live Filtering is a tool to help users fine-tune their search results - it's not a security feature or admin configurable filter. But my answer is a strong 'yes' if I answer your question not on the feature but the use-case level. To do what you're asking about, catalog Live Filtering would be used. An admin can define and preset dynamic, rule based permissions and assign that to individual users or groups - a very powerful and addressing many different aspects of security and workflow needs (Figures 3 and 4).

Catalog Live Filter works like a dynamic, rule based permission and is set up by the admin. Users will only see approved assets

(or any other subset defined by the filter setting) and will not even know that other records exist in the database.

User Live Filter can instantly be configured and selected from the user based on her/his current work focus

MM: Okay, let's move on to new features for using assets.

UK: Wait, before we do, there's one more thing to talk about with regard to searching. The Cumulus 8 Quicksearch tool - which works similar to an Internet search engine - has been updated. Users can now broaden or reduce the search Quicksearch results.

MM: So it allows you to do a search within search results?

UK: Yes, but it also enables you to add asset records to the current collection by adding additional search terms to the equation.

MM: So, it basically lets me add and subtract records from the collection just by adding new terms?

UK: That's exactly right.

MM: Once I fine-tune my collection, can I save the ultimate query that found them all? UK: You can save the collection, itself, which would be a shortcut to saving the search parameters. You could also create and even 


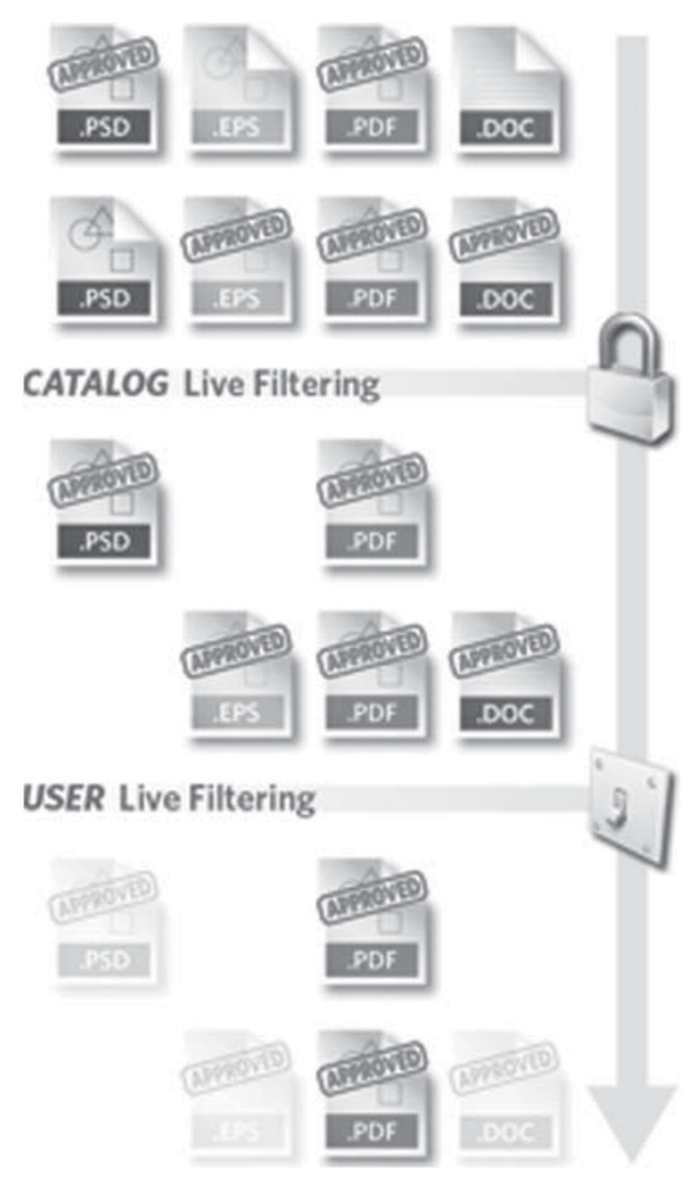

Figure 3: User Live Filtering enables users to define metadata conditions that are overlaid over search activity. This image illustrates the difference between the Catalog Live Filter and the User Live Filter.

share a record query that is based on your Quicksearch steps that could be used by others. MM: Okay, say my cataloged is configured, presumably by an administrator, and my assets are cataloged, perhaps by a catalog editor. What new, if anything, does Cumulus 8 offer regular users?

UK: Users asked for a way to compare assets side by side, so we're giving them that in Cumulus 8 , and more. The new Cumulus 8 Full screen Preview enables the user to preview up to six assets at a time. The user selects the assets and, once Full screen Preview is enabled, the user's entire screen becomes like an interactive light box - the Cumulus Client window and all other applications temporarily disappear. Inside the Full screen Preview, the user can drag and zoom within each preview section, and even quickly access an information view to edit metadata.

MM: Can I make notes while I'm previewing the assets?

UK: Actually, yes, and that's new for Cumulus 8 too. While you're viewing an asset, you can add little sticky notes to enter comments wherever you need. Or, you could draw rectangles around areas and comment on those.

MM: And these annotations are then saved with the asset?

UK: Actually, Cumulus saves the comments inside the asset record itself, so they become part of its metadata. This is great, because you can find assets based on searching for comments they contain. You can add more later, or delete existing ones - anything you need.

MM: Other than comments, what annotation options are available?

UK: With the initial release of Cumulus 8 , only sticky notes and rectangles are included. But underlying architecture changes to our database will enable us to take this feature much further in the future, if customers request it (Figure 5).

MM: Can you expound on these database changes?

UK: Sure. In a nutshell, we've created a new metadata field type that enables customers to define their own complex data types. Basically, it's a metadata field that can contain other metadata fields - a hierarchical data structure. This sounds more complicated than it is, though.

Imagine it this way, using Cumulus 8 annotations as an example. In addition to the comments themselves, users expect an annotation to contain the name of the commenter and a time stamp. These are three different metadata values that can now be handled as a single metadata entity now, thanks to this new data type. Plus, each asset record can contain zero or more of these entries.

MM: Sort of how an address book permits the addition of additional contacts, each based on shared set of field values?

UK: That's right.

MM: What are some of the reasons a customer would need to define a field like this in his DAM?

UK: We often say that Cumulus is useful for tracking an asset's history and future. What we 


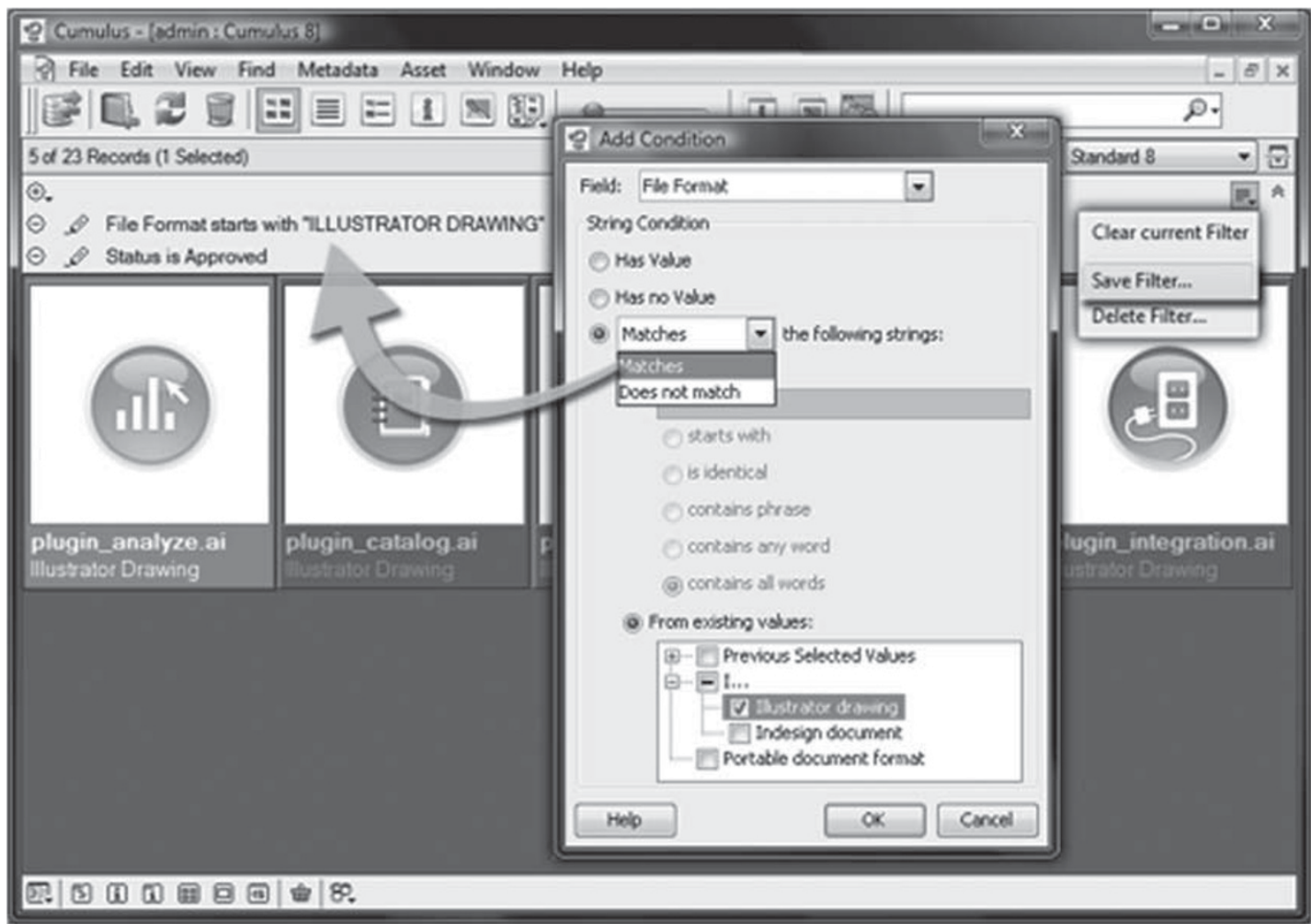

Figures 4: User Live Filtering enables users to define metadata conditions that are overlaid over search activity. This image illustrates the difference between the Catalog Live Filter and the User Live Filter.

mean by this is that much of what needs to be a tracked about an asset is variable. For example, when an asset is licensed for use, who licensed it? Previously, you could add all this information to a text field, but now you can define specific fields that are designed for the information you want to track, such as customer name, contact info, license expiration date, and each asset can have one or more of these data sets, which makes sense when you consider that the number of licensees you might have is infinite.

Now, using Cumulus 8, you could, for example, easily see which licenses are expiring soon and contact those customers for renewal. Just by searching for due dates that are, say, within a month, you cannot only see the assets themselves, but you can see the customer contact info.

MM: Couldn't you have done this by using categories, creating one category per customer and assigning assets that way?

UK: Yes, kind of - but keep in mind that it's not uncommon for users who have permissions to make metadata entries to not have permission to create and edit categories. Many sites see categories as a taxonomy or catalog structure issue they don't want users messing with on a day-to-day basis.

Plus, this opens up an entirely new paradigm with regard to how users can start tracking their metadata. For example, what in Cumulus 7 was a simple comments field can now be, in Cumulus 8, a comments history field. This makes inter-catalog communication between users easy, trackable and archivable too. And, of course, searchable. Email is great for general communications, but communicating about an asset inside the asset's record is better, because the entire correspondence stays right where it belongs - alongside the asset.

MM: I see. So, in many respects, this is metadata not necessarily about the asset, but about the workflow surrounding the asset. UK: Exactly. This is a huge improvement for Cumulus 8 that will largely go unnoticed at first, but I plan to do everything I can to spread the word! And, honestly, you've seen only the start of the power of this new architecture with the initial release of Cumulus 8 . 


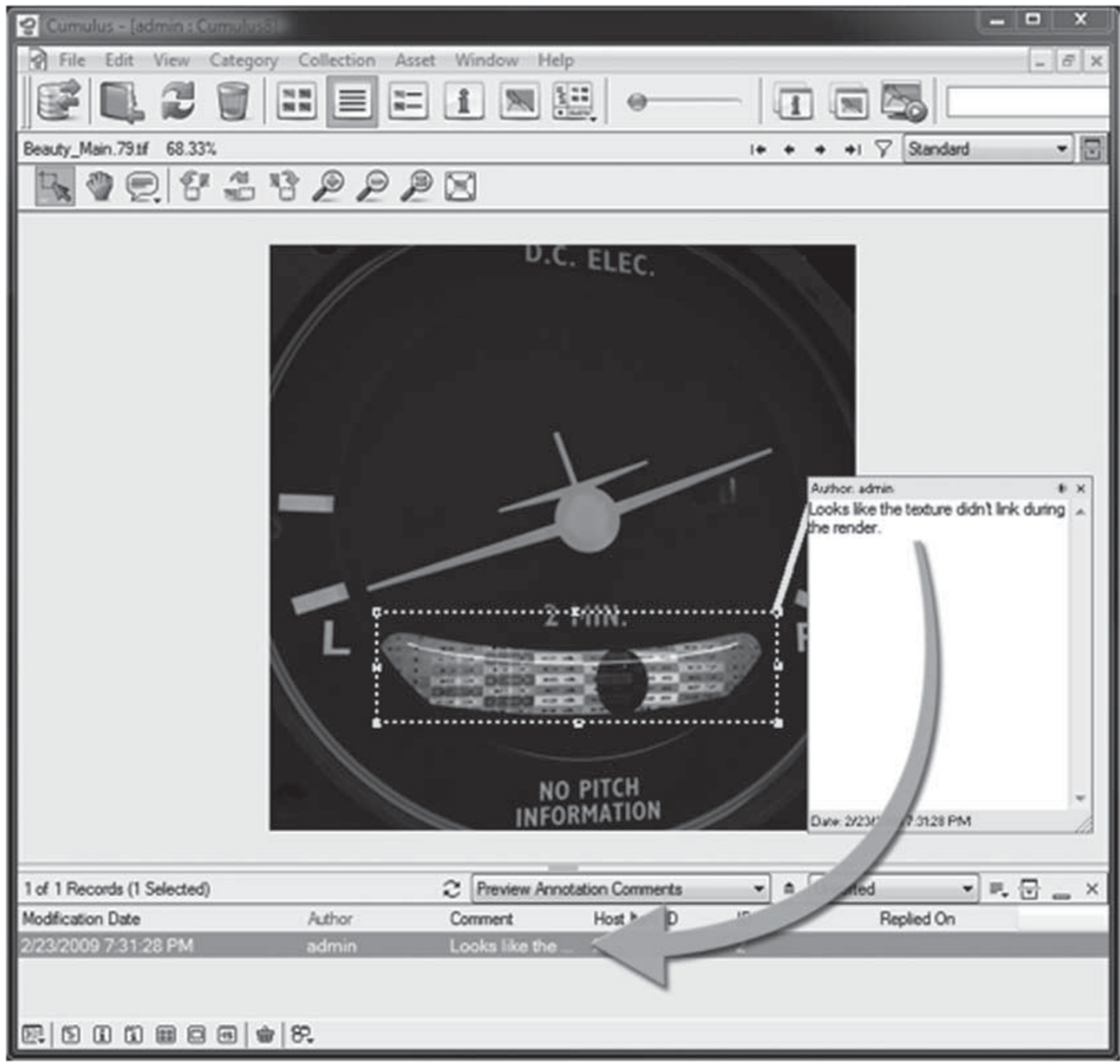

Figure 5: Annotations can be added inside the Preview window. They are stored with the asset record, and their contents are searchable, enabling you to find assets based on comments they contain. The Cumulus 8 window can be split to show a list of annotations for the selected asset record. User can search for comments via QuickSearch or Find window.

MM: An underdog for faceless technologies, are you?

UK: I do have a history of that, yes! For example, the field formulas released with Cumulus 7.5 got lots of blank stares at first, because customers weren't sure what to do with them. But I jumped right on them, because I saw how powerful they could be. Now, new features in Cumulus 8 make field formulas even more powerful than they were in Cumulus 7 . When users look at Cumulus 8, and then take a look at what's been introduced since Cumulus 7.0, they'll see that we've actually been building up to Cumulus 8 all this time. Much of the underlying power introduced in the various updates to Cumulus 7 - some of which seemed to lie dormant - becomes even more useful now. And, in many cases, it those technologies serve as the bedrock for what we've packed into 8 . MM: Why not just save everything up for a single release?

UK: Well, there are a few reasons we let Cumulus 8 technologies 'leak' into Cumulus 7 . For one, they were useful on their own, so there was no reason to hold them back from customers who could be benefiting from them all this time. But, by seeding some stuff along the way, we make the transition to Cumulus 8 
easier for users - and safer too - because we know the 'bedrock' we're now relying on in 8 has been tested and tweaked.

MM: Let's move on to other new things. Earlier, I saw you demoing an image-editing tool. That's new, correct?

UK: What you saw is called the Cumulus 8 Image Editor. Users select an asset and, when they open the Image Editor, a window appears that includes various tools from the Cumulus Pixel Image Converter, such as blur, sharpen, crop, etc.

What's great about the Image Editor, is that it performs its magic by editing previews of the assets, not the actual assets themselves (Figure 6). Not only does this keep assets safe, but it enables users to work with any asset that can be previewed in Cumulus, such as PDFs and PowerPoint presentations.

MM: So I can blur and crop a PDF?

UK: Yes, if you wanted to! Since Cumulus 7.6, Cumulus has been able to generate highresolution previews of PDF files natively, so you could use the Image Editor to blur, flip, convert -to grayscale or anything else you wanted to do with a PDF. You could even try something more functional, such as creating a series of website-ready thumbnails of your entire PDF or PowerPoint collection.

MM: So how does the Image Editor differ from the Pixel Image Converter? Why would someone use this instead?

UK: Conversions and such performed by the Pixel Image Converter are wrapped into actions that remain static, meaning once they are configured, they do specific things only. While this makes common processing options easy for users - they just select a menu option - many users wanted a more interactive way to finetune the effects of the Pixel Image Converter. Now, they can open the image and play with it all they like. When they get it just right, they can save it locally.

MM: All this, without the need for Photoshop. UK: Right, and that's a very important point, because it's common for users who are not designers to need variants of existing images. For example, a say press outlet asks me for a face shot of Michael Moon for an article. I've got a high-resolution Photoshop file of Michael Moon in my catalog, but it's a full body shot. My options are to either download the potentially massive Photoshop file - and hope I have Photoshop on my computer and that I

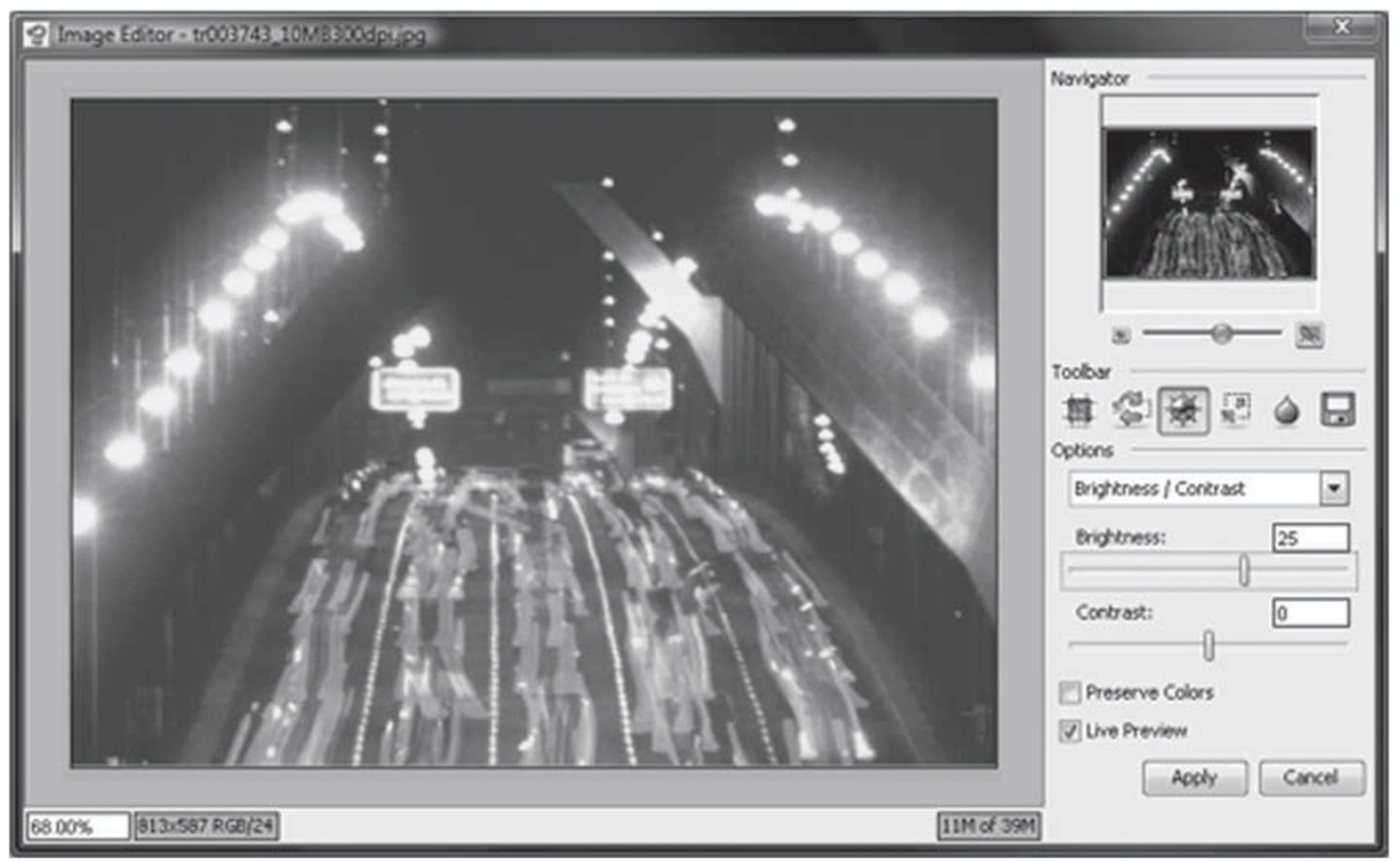

Figure 6: Cumulus 8 includes an image editor that enables users to crop, blur, adjust and otherwise manipulate assets. They can save their edits locally, and the original asset is never affected. 
know how to use it! - or I can go in and get exactly the crop job I need, without ever leaving Cumulus.

MM: So, this would mean interactive ad hoc renditions that include cropping and some light retouching could even be done by the news outlet itself?

UK: That's true, because the Image Editor is also available from within the Cumulus Web Client. A poor new agency photo editor could have an art director there barking over his shoulder, saying, 'Crop in a bit! Sharpen it! Darken it!' Once the edit was perfect, the news organization has exactly what they need.

MM: And you've made this possible, presumably, without needing to give them download access to the original file?

UK: That's correct, and it's a great example of what I was talking about when I mentioned how new Cumulus 8 technologies work with existing Cumulus technologies to make entirely new things possible - we've always been able to limit access to the original asset, but now look how much more useful the system becomes, even while those security measures are still in place.

MM: Tracking and statistics are listed as being new in Cumulus 8. What are you offering in that regard?

UK: Cumulus 8 can track events that occur to an asset from within Cumulus, such as when it was opened, previewed, emailed, checked-out, edited, etc. What's logged is up to the catalog admin.

MM: So, each time, for example, I preview an asset, my user name is recorded along with, presumably a time stamp of when the preview occurred?

UK: Assuming the catalog is configured to capture those events, yes. This is another example, in addition to annotations, where we're making use of our new hierarchical metadata field type. So, not only is all this information tracked, Cumulus 8 can even generate reports based on it.

MM: What sorts of reports are we talking about?

UK: The Cumulus Scheduler can examine a catalog at any interval and generate text reports and graphical charts of any or all of these events (Figure 7). This is great for being able to track who did what, but it's also useful for determining which of your assets are never used, and which you could be valuing higher than you are. Plus and marketing gurus love this! - you can actually test your metadata now.

MM: How so?

UK: Say, for example, you use the keyword 'airplane' to describe your aviation assets for 1 month, then you use 'aircraft' the next month. Cumulus 8 reporting helps you determine which term was more widely used, because on the use of the asset. If it was accessed more one month than the other, you have a better sense of how users think.

MM: This is same game marketeers play with Google Adwords - figuring out which words work best. Right?

UK: That's right, and it's easy to parallel that with what we're doing by thinking of the website as the cataloged asset. Which metadata terms are users using to find it? But it also makes even more basic marketing operations available, such as tracking the downloads of assets you've advertised. In that case, you are valuing your campaigns in addition to your assets.

MM: Yes, but let's be realistic: Marketing professionals are not going to rely on Cumulus for tracking their campaigns when tools designed for this use are available. Right? UK: We think they will! Certainly, any serious marketeer will not rely solely on Cumulus 8 for campaign tracking, but consider this scenario: Say, you sell clothing online and your catalog is based on assets retrieved from Cumulus. You run an add on MySpace advertising a particular item. Users see the ad, click on it and they're taken to your site. Okay, so Google, for example, will show you how many hits you got that day, and from where they came. But Cumulus 8 can also help you determine the user's level of interest in the item. Did she preview it? Did she preview other assets? Google can't help you once the user gets inside a database-driven environment like this. Cumulus 8 can!

MM: Canto released its partner marketplace on your website last year. What can you tell us about your partners and products they offer?

UK: The Canto website marketplace is a central resource for information on Cumulus products and add-ons published by our developer partners. It's the first time we've offered such a 


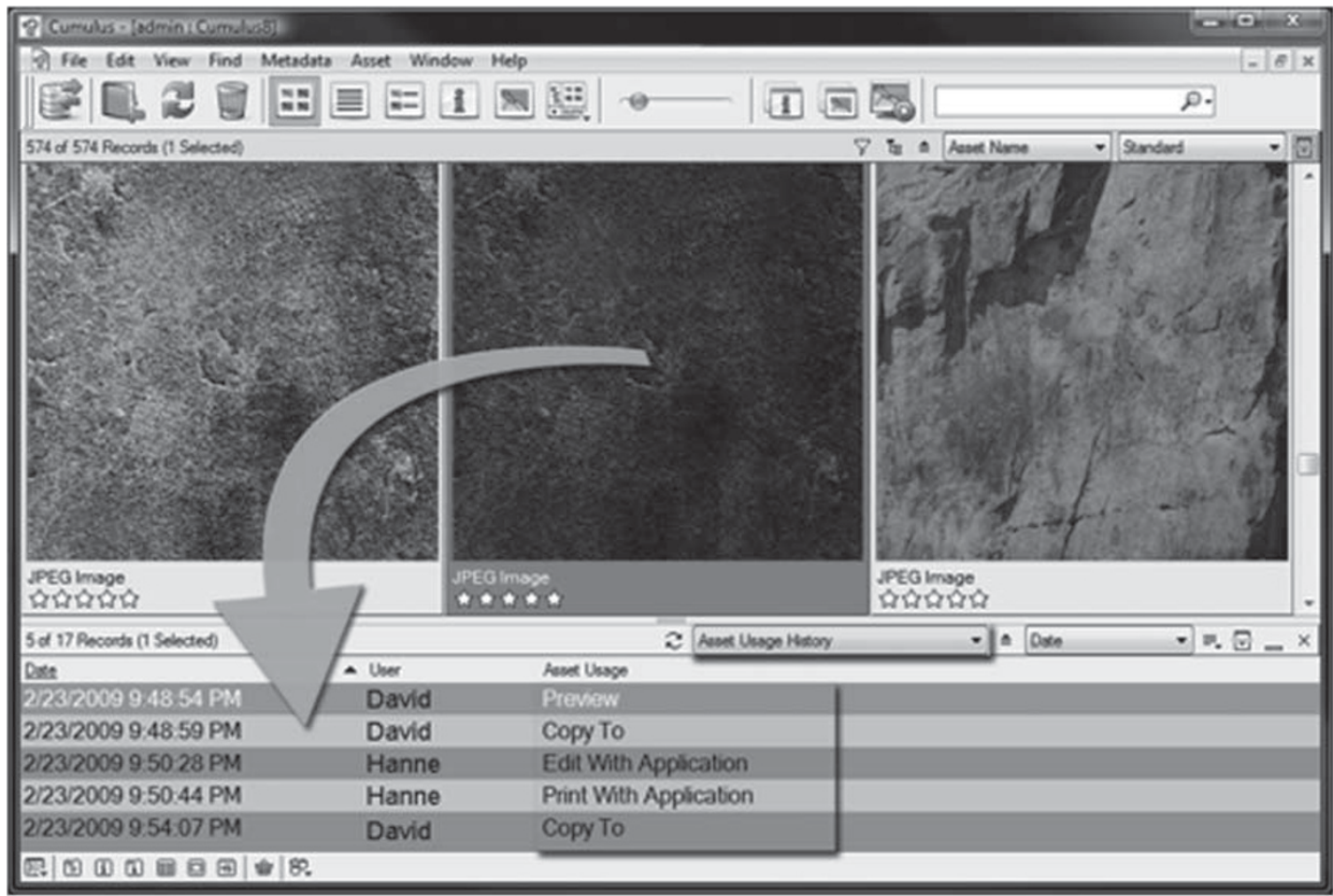

Figure 7: User events, such as previews, asset copying, printing, etc can be tracked by Cumulus 8. It's easy to see a list of events in the asset record view window, and reports can be based on the events too.

resource, and it was high time, considering the number of Cumulus enhancement products that are out there now.

MM: Is this an exhaustive list of partners, or are only certain partners featured?

UK: It's important to keep in mind that the marketplace is a list of products, not partners. The companies that make those products are Canto development partners, but Canto also has sales and integration partners, and strategic partners, such as Quark, Adobe, etc, too Sales and integration partners sell Cumulus, and might offer installation and integration services, but they don't offer actual products for sale, so you won't find them in the marketplace. They are listed elsewhere on the website, though.

MM: What sorts of products are common to find there?

UK: I don't think you'll find any particular type of product to be common, because so many of them are developed to fit a unique need. You'll see everything from eCommerce modules that plug into the Cumulus Web Clients, to entirely new Web Clients not based on Canto's Web Clients at all. There are other examples of alternatives to Canto technologies too, such as watermarking modules and metadata importing and exporting products.

MM: What limitations are there with regard to what partners can develop for Cumulus? How advanced could one of these products become? UK: We make the same Cumulus API that we use internally available to our development partners, so there's no much we can do to extend Cumulus that they can't do too. I think this is one of the reasons these companies chose Cumulus as a development platform - unlike many other DAM systems, we don't limit their potential. And, of course, we offer developers the largest installed user base in the world, which I suppose helps.

MM: I'd like you to talk to us about the development frameworks Canto offers. Specifically, give us a little bit of an overview of EJP, ESP and the C++ libraries.

UK: EJP stands for Embedded Java-based Plugins. We introduced EJaPs in previous versions of Cumulus as a means for replacing scripting languages, such as AppleScript, that did not support cross-platform processes. 
MM: So, you no longer support AppleScript in Cumulus?

UK: Correct. It just did not make sense for Canto to support proprietary OS technologies, especially when we can support a much better option based on Java. Anyone can develop a EJaP just once, and run on either the Mac or PC Cumulus Clients. Plus, we don't need to worry that Apple or whoever will change something in an OS update that will kill everyone's scripts.

MM: Yes, but you must have had some resistance from AppleScript fans?

UK: Initially, of course, but keep in mind that EJaPs have been in use since Cumulus 6, so anyone that was upset has long since gotten over it! In fact, our developer community has really grown to love EJaPs for a good reason: much better performance. In fact, many of the UI elements in the Cumulus Native Clients are based on EJaPs - our metadata pre-filler and Web Album are two examples that come to mind. You wouldn't be able to provide crossplatform solutions like those using AppleScript.

MM: Do EJaPs offer developers any mechanisms for serializing or otherwise protecting their products?

UK: Yes, then can be registered as part of the Cumulus installation, which is a wonderful benefit for partners. They can serialize their products. They can limit access to a certain number of licenses, which helps their business models.

MM: But EJaPs have to be run on the client side, right? There's no server version?

UK: Our ESP technology, which stands for embedded server plug-in, is our Cumulus Server equivalent. This provides developers with the same sort of cross-platform power on the back end that EJaPs provide inside the Client. MM: And there you're also running on Linux and Solaris in addition to Mac and Windows. I assume ESPs work on all?

UK: Yes, which is the wonderful thing about a Java-based environment. Plus, Java developers are easy to find, so a customer can even create some customization of their own systems.

MM: Would a customer have access to the various APIs and developer resources? UK: Yes, in fact we have many customers in the Canto Developer Program (CDP) doing this exact thing.
MM: So, there is an actual program for Cumulus development?

UK: Yes. Membership gets any member partner or customer - access to the APIs and other resources they need to develop Cumulus enhancements for sale or internal use.

MM: If the various Java-based APIs provide access to so much of Cumulus, what's the need for the $\mathrm{C}++$ libraries?

UK: High-level UI and metadata calculations are handled fine through Java, but when get down to the level of the operating system, you will need to start speaking $\mathrm{C}++$.

MM: Can you offer examples of when this is needed?

UK: The Cumulus filters, which we use to extract metadata from files, are all based on the $\mathrm{C}++$ libraries. So, if a customer or partner wanted to provide enhanced metadata support for some file format we don't support natively, they'd do that using the $\mathrm{C}++$ libraries. Also, if they wanted to create a new asset storage module - the modules we use to read and write data - they'd have to do that with this library.

MM: Would it be fair to say that when interfacing with humans, Cumulus does that via Java; but when interfacing with the hardware, it uses $\mathrm{C}++$ ?

UK: That's true in most cases, but ESPs work on the server without interfacing with humans. I think it's more accurate to say that when Cumulus needs to peer inside a file or storage medium, it uses $\mathrm{C}++$ to do so.

MM: Is use of the $\mathrm{C}++$ library as common as the Java libraries?

UK: Definitely not. Everyone knows Java, so even if an actual product isn't planned, you see Java development taking place, because it's so easy by comparison.

MM: Is EJaP an industry standard or broadly accepted framework? Or does everyone kind of do their own little version?

UK: The Java technology is standard. We're not doing anything magical with Java that anybody that knows Java wouldn't be able to do. But the API is obviously unique to Cumulus. But any Java developer can come and start developing EJaPs and ESPs pretty much straight away by just looking at the API. The Cumulus-published APIs are not overly complicated.

MM: And the ESPs are the same. 
UK: Yes.

MM: And what about the $\mathrm{C}++$ libraries? Are they standard libraries?

UK: Again, the API is Cumulus-specific, but anyone familiar with $\mathrm{C}++$ development will be able to get what they need from it pretty quick. MM: Overall, how capable are all these frameworks?

UK: Look at it this way, Canto uses these very same APIs for our own development, so there's lots of potential!

MM: If would be developers needed training, what resources are available to them?

UK: Once they join the CDP, they have access to a number of resources, including documentation, other developers and training through Canto Professional Services (CPS).

MM: Yes, I'm a bit confused about CPS. This was launched last year, yet it seems as though Canto has been offering these services for years. UK: Canto Professional Services was formalized into a department last year, but you are correct, Canto has been doing this stuff for many years. CPS provides classes for users, admins and developers, in addition to providing installation and integration services worldwide.

MM: How does Canto reconcile this with partners who provide the same services? Isn't this seen as competition?

UK: It doesn't turn out that way, because the world is pretty big - first off - but even in areas of overlap, the network of options tends to be more partner-like than competitive. For example, there are times when a work order comes into Canto and we know it would be better suited toward a partner. So, assuming the customer is okay with it, we'll connect them up.

MM: When would there ever be a situation in which a partner was better equipped to handle service than Canto?

UK: Typically it's more about logistics than know how. Obviously, the folks in CPS are pretty capable when it comes to Cumulus. But, they don't speak every language on Earth, nor can they cost-effectively be in every corner of the world. So, for example, if we get a request for a big installation in some part of the world where we have a fantastic partner, it's usually better for everyone if that partner does the work. They speak the language, and they're available for longer-term needs.

MM: What about those partners who are nearer the Canto offices? Don't they get the short end of that stick?

UK: Canto offices are in Berlin and San Francisco and, believe me, neither place has a shortage of customers! In fact, the partners nearby us are already taking advantage of the lucrative local markets, so they usually have their hands full already. But even still, if a call comes in that nicely fits a particular partner's area of expertize, we're happy to transfer the business. Plus, we sometimes encounter customers who would rather deal with Canto directly, and we sometimes encounter others who prefer the, shall we say, unbiased perspectives of a partner.

Ultimately, this is about making customers happy.

MM: And you're confident this Canto/partner hybrid network is doing that?

UK: Not always - I'll admit that. But most of the time, yes. And, for those times when some extra customer attention is needed, I've yet to see Canto or one of our partners hesitant to get involved to make things right for the customer.

And when you consider that we've sold well over 13500 Cumulus Servers in the past 10 or so years, we have definitely got most of it right! With regard to Canto, our partners and our customers - I'd say we're all lucky to have one another. And I think most of us would agree on that!

MM: A great summary. Thank you!

UK: And thank you for the great questions and the opportunity to answer them! 